\title{
Debreu's coefficient of resource utilization, the Solow residual, and TFP: the connection by Leontief preferences
}

\author{
Thijs ten Raa
}

Published online: 25 September 2008

(c) The Author(s) 2008. This article is published with open access at Springerlink.com

\begin{abstract}
Debreu's coefficient of resource utilization is freed from individual data requirements. The procedure is shown to be equivalent to the imposition of Leontief preferences. The rate of growth of the modified Debreu coefficient and the Solow residual are shown to add up to TFP growth. This decomposition is the neoclassical counterpart to the frontier analytic decomposition of productivity growth into technical change and efficiency change. The terms can now be broken down by sector as well as by factor input.
\end{abstract}

Keywords Efficiency - Productivity ·

Debreu's coefficient - Solow residual

JEL Classifications $\quad$ D24 $\cdot$ O47

\section{Introduction}

Total factor productivity (TFP) may grow by more efficient utilization of resources or by technical change. Debreu (1951) measured the utilization of resources and Solow (1957) measured technical change, but their models are remote. Solow's model is macro-economic and assumes perfect competition, while Debreu's model is micro-

This paper is an offspring of the workshop Alternative measures of total factor productivity growth held on 13 September 2002 at the Department of Econometrics and Operations Research of Tilburg University and co-sponsored by the Netherlands Organization for Scientific Research (Grant 460-02-031-A).

T. ten Raa ( $₫)$

Tilburg University, Box 90153, 5000 LE Tilburg,

The Netherlands

e-mail: tenRaa@UvT.nl economic and assumes no technical change. In this paper, I show how the measures of Debreu and Solow can be commingled into TFP. I take Debreu's model as point of departure, because it is quite general and, therefore, accommodating. The drawback of Debreu's coefficient of resource utilization, however, is that it hinges on individual preferences data. I will free his coefficient from this prohibitive data requirement, by making Debreu's concept of a 'better' commodity set independent of the specifics of individual preferences. The procedure will be shown to be equivalent to the adoption of Leontief preferences, confirming Diewert's (1983) idea that such preferences remove misallocations between consumers as a source of inefficiency. The consequent coefficient of resource utilization yields a more conservative estimate of inefficiency than Debreu's coefficient resource of utilization. As a bonus, the procedure makes the measure of inefficiency a function of total consumption only, not the individual breakdown. This paves the way for macro-economic applications and Solow residual analysis. The Solow residual is generalized to Debreu's setting.

Neoclassical economics encounters some refreshing competition from frontier analysis. See Nishimizu and Page (1982), Färe et al. (1994), Färe and Grosskopf (1996), Kumar and Russell (2002) and the references given there. This literature provides a useful decomposition of productivity growth into technical change and efficiency change. I take the idea into the neoclassical general equilibrium realm. The connection is at a rather abstract level, for the mechanisms behind efficiency change are different in frontier analysis and neoclassical economics. Frontier analysis captures technological catch-up with the leader and the choice of inputs in terms of costs. Neoclassical analysis captures potential reallocations of resources between sectors. This type of efficiency change is harder to 
detect. Frontier analytic inefficiency is exposed by the gap with the best practice, a conceptually straightforward concept. Neoclassical inefficiency, however, not only comprises gaps with production possibility frontiers, but also hidden misallocations. A nice exposition is Debreu (1951) and Diewert (2001) discusses the reduction to TFP. A contribution of my paper is that it shows how the tools of frontier analysis, particularly the input- and output-distance functions, can be applied to the measurement of allocative efficiency.

The pieces of the puzzle fit pleasingly well. More precisely, in this paper I show that TFP growth is the sum of technical change and efficiency change, where the former is the (generalized) Solow residual and the latter is the rate of growth of the modified coefficient of resource utilization.

\section{The Debreu coefficient of resource utilization}

Debreu (1951) measures the inefficiency of the allocation of resources in an economy by calculating how much less resources could attain the same level of satisfaction to the consumers. I will review his so-called coefficient of resource utilization.

The economy comprises $m$ consumers with preference relationships $\succsim_{i}$ and observed consumption vectors $\mathbf{x}_{i}^{0} \in \mathbb{R}^{l}$ $(i=1, \ldots, m)$, where $l$ is the number of commodities. $Y \subset$ $\mathbb{R}^{l}$ is the set of possible input vectors (net quantities of commodities consumed by the whole production sector during the period considered), including the observed one, $\mathbf{y}^{0}$. A combination of consumption vectors and an input vector is feasible if the total sum-the economy-wide net consumption-does not exceed the vector of utilizable physical resources, $\mathbf{z}^{0} \in \mathbb{R}^{l}{ }^{1}$ Vector $\mathbf{z}^{0}$ is assumed to be at least equal to the sum of the observed consumption and input vectors, ensuring the feasibility of the latter.

The set of net consumption vectors that are at least as good as the observed ones is

$\mathcal{B}=\left\{\sum \mathbf{x}_{i} \mid \mathbf{x}_{i}{ }_{i} \mathbf{x}_{i}^{0}, i=1, \ldots, m\right\}+Y$

The symbol $\mathcal{B}$ stands for 'better' set. The minimal resources required to attain the same levels of satisfaction that come with $\mathbf{x}_{i}^{0}$ belong to $\mathcal{B}^{\mathrm{min}}$, the South-western edge or subset of elements $\mathbf{z}$ that are minimal with respect to $\geqq$. ${ }^{2}$ Assume that preferences are convex and continuous, and that production possibilities form a convex and closed set,

\footnotetext{
${ }^{1}$ For example, if the last commodity, $l$, represents labor, and this is the only nonproduced commodity, then $\mathbf{z}^{0}=N e_{l}$, where $N$ is the labor force and $e_{l}$ the $l$-th unit vector.

${ }^{2}$ By convention, this vector inequality holds if it holds for all components.
}

then the separating hyperplane theorem yields a supporting price vector $\mathbf{p}(\mathbf{z})>\mathbf{0}$ such that $\mathbf{z}^{\prime} \in \mathcal{B}$ implies $\mathbf{p}(\mathbf{z}) \cdot \mathbf{z}^{\prime} \geqq \mathbf{p}(\mathbf{z}) \cdot \mathbf{z}^{3}{ }^{3}$ The Debreu coefficient of resource utilization is defined by

$\rho=\max _{\mathbf{z}}\left\{\mathbf{p}(\mathbf{z}) \cdot \mathbf{z} / \mathbf{p}(\mathbf{z}) \cdot \mathbf{z}^{0} \mid \mathbf{z} \in \mathcal{B}^{\min }\right\}$

Coefficient $\rho$ measures the distance from the set of minimally required physical resources, $\mathbf{z} \in \mathcal{B}^{\text {min }}$, to the utilizable physical resources, $\mathbf{z}^{0}$, in the metric of the supporting prices (which indicate welfare indeed). Debreu (1951, p. 284) shows that the distance or the max in (2) is attained by ${ }^{4}$

$\mathbf{z}=\rho \mathbf{z}^{0} \in \mathcal{B}^{\min }$

In other words, the Debreu coefficient of resource utilization is the smallest fraction of the actually available resources that would permit the achievement of the levels of satisfaction that come with $\mathbf{x}_{i}^{0}$. Coefficient $\rho$ is a number between zero and one, the latter indicating full efficiency. In modern terminology, this result means that $\rho$ is the input-distance function, determined by the program

$\rho=\min _{r}\left\{r \mid \sum \mathbf{x}_{i}+\mathbf{y} \leqq r \mathbf{z}^{0}, \mathbf{x}_{i} \succsim_{i} \mathbf{x}_{i}^{0}, \mathbf{y} \in Y\right\}$

There is also an output-distance function, but that one is opaque. The measurement of satisfaction is in terms of

${ }^{3 \mathbf{p}>\mathbf{0} \text { means }}$ that all components are positive. The prices are positive because of the min-superscript in $\mathbf{z} \in \mathcal{B}^{\min }$ and the fact that $\mathbf{z}$ is the only point in common to $\mathcal{B}$ and $\left\{\mathbf{z}^{\prime} \mid \mathbf{z}^{\prime} \leqq \mathbf{z}\right\}$; hence, $\mathbf{p}$ may be chosen such that $\mathbf{p} \cdot \mathbf{z}^{\prime}<\mathbf{p} \cdot \mathbf{z}$ for $\mathbf{z}^{\prime} \leqq \mathbf{z}$ (except $\left.\mathbf{z}^{\prime}=\mathbf{z}\right)$. This argument requires no monotonicity and Debreu (1951) does not assume it indeed. An example is an exchange economy $(Y=\{0\})$ with one consumer and two commodities of which the quantities are nonnegative. Let $\mathbf{x} \succsim \mathbf{x}^{\prime}$ if $\left(2-x_{1}\right)^{2} \leqq\left(2-x_{1}^{\prime}\right)^{2}$. Let $\mathbf{x}^{0}=\mathbf{z}^{0}=\left(\begin{array}{ll}3 & 1\end{array}\right)$. Then $\mathcal{B}^{\text {min }}=\left\{\mathbf{x} \mid \mathbf{x} \succsim \mathbf{x}^{0}\right\}^{\text {min }}=\left\{\mathbf{x} \mid 1 \leqq x_{1} \leqq 3\right\}^{\text {min }}=\left\{\left(\begin{array}{ll}1 & 0\end{array}\right)\right\}$. In this point, any positive price vector renders $\mathcal{B}=\left\{\mathbf{x} \mid 1 \leqq x_{1} \leqq 3\right\}$ more expensive. ${ }^{4}$ There are two, related caveats in Debreu's (1951) analysis: $\mathbf{z}=$ $\rho \mathbf{z}^{0} \in \mathcal{B}^{\min }$ need not exist and $\rho$ may not be unique if the separating price vector is not unique. Consider again the exchange economy $(Y=\{0\})$ with one consumer and two commodities. Let $\mathbf{x} \succsim \mathbf{x}^{\prime}$ if $\min \left(x_{1}, x_{2}\right) \geqq \min \left(x_{1}^{\prime}, x_{2}^{\prime}\right)$. Let $\mathbf{x}^{0}=\left(\begin{array}{ll}1 & 1\end{array}\right)$ and $\mathbf{z}^{0}=\left(\begin{array}{ll}1 & 2\end{array}\right)$. Then $\mathcal{B}^{\text {min }}=\left\{\mathbf{x} \mid \mathbf{x} \succsim \mathbf{x}^{0}\right\}^{\text {min }}=\left\{\mathbf{x} \mid \mathbf{x} \geqq\left(\begin{array}{ll}1 & 1\end{array}\right)\right\}^{\text {min }}=\left\{\left(\begin{array}{ll}1 & 1\end{array}\right)\right\} \quad$ contains no $\rho \mathbf{z}^{0}=\rho\left(\begin{array}{ll}1 & 2\end{array}\right)$. What is the coefficient of resource utilization? In this case, any $\mathbf{p}>\mathbf{0}$ separates $\mathcal{B}=\left\{\mathbf{x} \mid \mathbf{x} \geqq\left(\begin{array}{ll}1 & 1\end{array}\right)\right\}$ and $\left\{\mathbf{z}^{\prime} \mid \mathbf{z}^{\prime} \leqq\left(\begin{array}{ll}1 & 1\end{array}\right)\right\}$; hence (2) yields $\rho=\mathbf{p} \cdot\left(\begin{array}{ll}1 & 1\end{array}\right) / \mathbf{p} \cdot\left(\begin{array}{ll}1 & 2\end{array}\right)=\left(p_{1}+p_{2}\right) /\left(p_{1}+2 p_{2}\right)$, a number between 0.5 and 1 . To resolve the multiplicity, we may address the efficiency problem in primal space. The preference relationship is represented by utility $\min \left(x_{1}, x_{2}\right)$. Subject to feasibility constraint $\mathbf{x} \leqq \mathbf{z}^{0}=\left(\begin{array}{ll}1 & 2\end{array}\right)$, the maximum utility is 1 . This is attained by $\mathbf{x}^{0}=\left(\begin{array}{ll}1 & 1\end{array}\right)$. Hence the allocation is optimal. Following Debreu's (1951, p. 275) introduction, $\rho=1$. This implies that $\mathbf{p}=(10)$. Indeed, this is the supporting price system of the second welfare theorem. However, it is not positive.

If the minimal $\rho \mathbf{z}^{0}$ belongs to $\mathcal{B}^{\text {min }}$, then the prices in (2) are positive and the coefficient $\rho$ generated by (4) solves (2), following Debreu (1951, p. 284). If the minimal $\rho \mathbf{z}^{0}$ does not belong to $\mathcal{B}^{\text {min }}$, the prices in (2) are only nonnegative, but the coefficient $\rho$ generated by (4) still solves (2). 
utility, an ordinal concept that generally admits no aggregation over consumers.

\section{The Farrell efficiency measure}

Following ten Raa (2007), let me clear up some confusion on the relationship between Debreu and Farrell's efficiency measures. Farrell (1957) decomposes efficiency in technical efficiency and allocative efficiency. He notes the similarity between his technical efficiency and the Debreu coefficient of resource utilization. Indeed, both the Farrell technical efficiency measure and the Debreu coefficient of resource utilization are defined through proportionate input contractions. The analogy is sheer formality, but confusing at a conceptual level. It suggests that Farrell takes the Debreu coefficient, augments it, and thus constructs a more encompassing overall measure. It is the other way round; the sway of the Debreu coefficient is far greater than that of Farrell's measure. Particularly Farrell's allocative efficiency measure is a partial (dis)equilibrium concept, conditioned on prices. It takes into account the cost reduction attainable by changing the mix of the inputs, given the prices of the latter. The Debreu coefficient, however, is a general (dis)equilibrium concept. It measures the technical and allocative inefficiency in the economy given only its fundamentals: resources, technology, and preferences. Prices are derived and enter the definition of the Debreu coefficient, see (2). Debreu (1951) then proves that the coefficient can be freed from these prices, by Eq. 3 or nonlinear program (4). Prices remain implicit, however. They support the better set in the point of minimally required physical resources. The Debreu coefficient measures technical and allocative inefficiency, both in production and consumption, solving the formidable difficulty involved in assessing prices, referred to by Charnes et al. (1978, p. 438). Farrell refrains from this, restricting himself to technical efficiency and price-conditioned allocative efficiency.

The formal analogy between the Debreu coefficient and the Farrell measure of technical efficiency prompted Zieschang (1984) to coin the phrase "Debreu-Farrell measure of efficiency," a term picked up by Chakravarty (1992) and Grifell-Tatjé et al. (1998). This practice is confusing. Debreu's coefficient of resource allocation encompasses both Farrell's technical efficiency and his allocative efficiency measures, and frees the latter from prices. On top of this, Debreu's coefficient captures consumers' inefficiencies. The confusion persists. Färe et al. (2002) speak of the "Debreu-Farrell measure of technical efficiency." A recent review of Farrell's contribution states

"(Debreu) worked only from the resource cost side, defining his coefficient as the ratio between minimized resource costs of obtaining a given consumption bundle and actual costs, for given prices and a proportional contraction of resources." Førsund and Sarafoglou (2002), footnote 4.

However, Debreu (1951) calculates the resource costs not of a given consumption bundle, but of an (intelligently chosen) Pareto equivalent allocation. (And the prices are not given, but support the allocation.) It is true, however, that the Debreu measure would become applicable if the aggregated consumption bundle can be considered given. The next section shows that this pragmatic approach is doable. The approach is exact if preferences are Leontief, as the subsequent section will prove.

\section{Absent individual data: the Debreu-Diewert coefficient of resource utilization}

Following Debreu (1951) a simple symbol $\mathcal{B}$ has been used to denote the 'better set.' Definition (1) reveals, however, that the set depends on the observed consumption vectors and on preferences. The informational requirements involved are prohibitive. To overcome this problem, I will define a version of the Debreu coefficient, namely the Debreu-Diewert coefficient of resource utilization, $\rho^{*}$. Only in the next section it will transpire why I choose this name.

I want that the notion of 'better' set is independent of the specifics of preferences. For this purpose, all I assume is that preferences are weakly monotonic in the sense that they belong to

$\mathcal{M}=\left\{\succsim \mid\left(\mathbf{x}^{\prime} \geqq \mathbf{x}\right)\right.$ implies $\left.\left(\mathbf{x}^{\prime} \succsim \mathbf{x}\right)\right\}$

I now define the tight better set as the intersection of all better sets over $\mathcal{M}$ :

$\mathcal{B}^{*}=\cap\left\{\sum \mathbf{x}_{i} \mid \mathbf{x}_{i} \succsim \mathbf{x}_{i}^{0}, i=1, \ldots, m\right\}+Y$

The replacement of the better set, $\mathcal{B}$, by the tight better set, $\mathcal{B}^{*}$, implies that definition (2) produces the DebreuDiewert coefficient $\rho^{*}$ instead of the Debreu coefficient $\rho$. A comparison between these two coefficients is obtained by rewriting program (4):

$\rho^{*}=\min _{r}\left\{r \mid \sum \mathbf{x}_{i}+\mathbf{y} \leqq r \mathbf{z}^{0}, \mathbf{x}_{i} \succsim \mathbf{x}_{i}^{0}\right.$ for all $\left.\succsim \in \mathcal{M}, \mathbf{y} \in Y\right\}$

The constraint set of (7) is contained in the one of (4); hence the solution to program (4) must be sharper:

$\rho \leqq \rho^{*}$

In other words, use of the Debreu-Diewert coefficient will overestimate efficiency hence underestimate inefficiency. Debreu's (1951) measure of inefficiency reflects scope for 


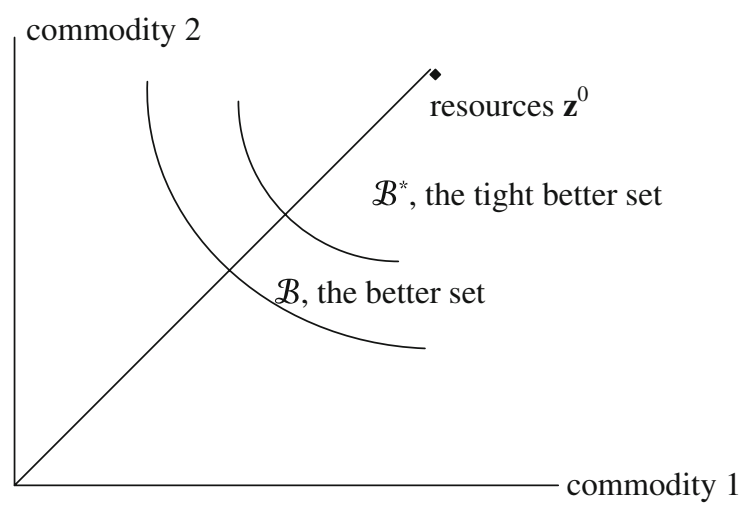

Fig. 1 Half the resources suffice to make consumers at least as well off. Two-thirds of the resources are needed to produce the total consumption vector. The Debreu coefficient of resource utilization is $1 / 2$ and the Debreu-Diewert coefficient is $2 / 3$

reallocation of resources between consumers with different tastes and, therefore, is quite high; the flipside of this observation is that the Debreu coefficient is relatively low. Figure 1 illustrates.

\section{The Debreu-Diewert coefficient and Leontief preferences}

I can be a bit more specific about the Debreu-Diewert coefficient of resource utilization. I will show that it is generated by Leontief preferences. As I noted in the introduction, Diewert (1983) first had the idea that such preferences remove misallocations between consumers as a source of inefficiency. This explains the name giving.

Leontief preferences $\succsim(\mathbf{a})$ with nonnegative bliss point $\mathbf{a} \in \mathbb{R}_{+}^{l}$ are defined for nonnegative consumption vectors by $\mathbf{x}^{\prime} \succsim(\mathbf{a}) \mathbf{x}$ if $\min x_{k}^{\prime} / a_{k} \geqq \min x_{k} / a_{k}$ where the minimum is taken over commodities $k=1, \ldots, l$. The minima exist if $\mathbf{a}$ is nonzero, which I assume. ${ }^{5}$ The following lemma shows that the tight better set is obtained by imposing Leontief preferences on all individuals. Notice, however, that the preferences feature the observed individual consumption baskets as bliss points and, therefore, differ.

Lemma $\mathcal{B}^{*}=\left\{\sum \mathbf{x}_{i} \mid \mathbf{x}_{i} \succsim\left(\mathbf{x}_{i}^{0}\right) \mathbf{x}_{i}^{0}, i=1, \ldots, m\right\}+$ $Y=\left\{\mathbf{x} \mid \mathbf{x} \geqq \sum \mathbf{x}_{i}^{0}\right\}+Y$.

Proof Using the fact $\succsim\left(\mathbf{x}_{i}^{0}\right) \in \mathcal{M}$, I have that the first term of $\mathcal{B}^{*}$ in (6), $\cap\left\{\sum \mathbf{x}_{i} \mid \mathbf{x}_{i} \succsim_{i} \mathbf{x}_{i}^{0}, i=1, \ldots, m\right\} \subset$ $\left\{\sum \mathbf{x}_{i} \mid \mathbf{x}_{i} \succsim\left(\mathbf{x}_{i}^{0}\right) \mathbf{x}_{i}^{0}, i=1, \ldots, m\right\}=\left\{\sum \mathbf{x}_{i} \mid \mathbf{x}_{i} \geqq \mathbf{x}_{i}^{0}\right\}=\{\mathbf{x} \mid \mathbf{x}$ $\left.\geqq \sum \mathbf{x}_{i}^{0}\right\} \subset \cap\left\{\sum \mathbf{x}_{i} \mid \mathbf{x}_{i} \succsim_{i} \mathbf{x}_{i}^{0}, i=1, \ldots, m\right\}$, which is itself. The last inclusion is shown as follows. For $\mathbf{x} \geqq \sum \mathbf{x}_{i}^{0}$, allocate the surplus in any nonnegative way, for example

\footnotetext{
5 Situations like labor supply are covered by letting the commodity be leisure time. Division by zero is assumed to yield infinity.
}

by putting $\mathbf{x}_{1}=\mathbf{x}_{1}^{0}+\mathbf{x}-\sum \mathbf{x}_{i}^{0}, \mathbf{x}_{2}=\mathbf{x}_{2}^{0}, \ldots, \mathbf{x}_{m}=\mathbf{x}_{m}^{0}$, then $\mathbf{x}_{i} \succsim_{i} \mathbf{x}_{i}^{0}$ for all $\succsim_{i} \in \mathcal{M}$. QED

The first equality in the Lemma implies that if the consumers have Leontief preferences, then the Debreu coefficient reduces to the Debreu-Diewert coefficient. The second equality in the Lemma, $\left\{\sum \mathbf{x}_{i} \mid \mathbf{x}_{i} \geqq \mathbf{x}_{i}^{0}\right\}=$ $\left\{\mathbf{x} \mid \mathbf{x} \geqq \sum \mathbf{x}_{i}^{0}\right\}$, is a perfect aggregation result. Aggregated consumption is monotonic if and only if individual consumptions are. One might say that if preferences are Leontief with varying bliss points (according to the observed consumption baskets), there is a social welfare function. The better set, $\mathcal{B}$, is freed from preferences, $\succsim_{i}$, as well as from individual consumption baskets, $\mathbf{x}_{i}^{0}$. The tight better set, $\mathcal{B}^{*}$, depends only on the total consumption vector, $\sum \mathbf{x}_{i}^{0}$. This modification facilitates measurement of the coefficient of resource utilization. In fact, the tightening creates the option to determine the degree of efficiency in terms of outputs, resurrecting the output-distance function.

Corollary Assume that the total consumption vector $\sum \mathbf{x}_{i}^{0}$ is nonnegative and nonzero. Assume that the production set $Y$ features the impossibility to produce something from nothing and constant returns to scale. Then $\gamma=1 / \rho^{*}$ transforms the input-distance function program (7) into the output-distance function program

$\gamma=\max \left\{c \mid c \sum \mathbf{x}_{i}^{0}+\mathbf{y} \leqq \mathbf{z}^{0}, \mathbf{y} \in Y\right\}$

Proof By the Lemma, program (7) can be rewritten as

$\rho^{*}=\min \left\{r \mid \sum \mathbf{x}_{i}+\mathbf{y} \leqq r \mathbf{z}^{0}, \mathbf{x}_{i} \succsim\left(\mathbf{x}_{i}^{0}\right) \mathbf{x}_{i}^{0}, i=1, \ldots, m, \mathbf{y} \in Y\right\}$

or

$\rho^{*}=\min \left\{r \mid \mathbf{x}+\mathbf{y} \leqq r \mathbf{z}^{0}, \mathbf{x} \geqq \sum \mathbf{x}_{i}^{0}, \mathbf{y} \in Y\right\}$

This can be simplified further to

$\rho^{*}=\min \left\{r \mid \sum \mathbf{x}_{i}^{0}+\mathbf{y} \leqq r \mathbf{z}^{0}, \mathbf{y} \in Y\right\}$

The solution is positive. (Otherwise input vector $\mathbf{y} \leqq$ $-\sum \mathbf{x}_{i}^{0} \leqq 0$, but not equal to zero, would produce something from nothing.) The transformation is completed by multiplication by $c=1 / r$ and a change of variable ( $c \mathbf{y}$ to y), using constant returns to scale. QED

The output-distance function program informs us by which factor the total consumption vector can be expanded, given the resources.

\section{Application to national accounts}

The corollary shows that under constant returns to scale the inverse of the Debreu-Diewert coefficient of resource 
utilization is the expansion factor of the economy, $\gamma$. ten Raa (2005) calculates $\gamma$ for the Canadian economy, defines $1-1 / \gamma$ as inefficiency, and decomposes the latter into productive inefficiency, allocative inefficiency, and trade inefficiency. It follows that ten Raa's (2005) measure of inefficiency is $1-\rho^{*}$. In view of inequality (8), this measure of inefficiency underestimates Debreu's (1951) degree of inefficiency, $1-\rho$. Debreu (1951) finds more scope for efficiency gains as marginal rates of substitution may be equalized across consumers. The Debreu-Diewert coefficient does not take into account this source of inefficiency.

ten Raa and Mohnen (2002) and ten Raa (2005) divide the commodities between produced goods and factor inputs, respectively. Use table $\mathbf{U}$ is a table depicting the use of goods by sectors and make table $\mathbf{V}$ is a table depicting the outputs of the sectors in terms of goods. $\mathbf{U}-\mathbf{V}^{\top}$ is the net input table; its dimension is that of goods by sectors. ${ }^{6} \mathbf{L}$ is the factor input table; its dimension is factor inputs by sectors. An element of $\mathbf{y} \in Y$ has components $\left(\mathbf{U}-\mathbf{V}^{\top}\right) \mathbf{s}$ and $\mathbf{L s}$, and $Y$ is defined by letting the allocation vector $\mathbf{s} \geqq 0$. Similarly, $\sum \mathbf{x}_{i}^{0}$ has components $\mathbf{f}$ and $\mathbf{0}$, where $\mathbf{f}$ is the vector of final goods consumption, while $\mathbf{z}^{0}$ has components $\mathbf{0}$ and $\mathbf{N}$, where $\mathbf{N}$ is the vector of factor endowments, and $\mathbf{z}$ has components $\mathbf{0}$ and Ls. The outputdistance function program of the corollary becomes

$\gamma=\max _{c, s}\left\{c \mid c \mathbf{f}+\left(\mathbf{U}-\mathbf{V}^{\top}\right) \mathbf{s} \leqq \mathbf{0}, \mathbf{L s} \leqq \mathbf{N}, \mathbf{s} \geqq \mathbf{0}\right\}$

The solution to this program yields the potential standard of living, relative to the observed one. ${ }^{7}$ The shadow prices of the second constraint yield the factor productivities.

The standard interpretation of a sector is that of an industry, but conceptually it is possible to disaggregate it into firms, for example by using the reported data. Then program (9) will select for each industry best practice firms, which transform the industry inputs into potential industry output, in the sense of Johansen (1972).

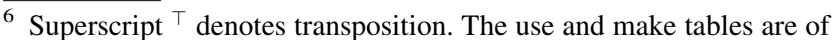
opposite dimension in the System of National Accounts (ten Raa, 2005).

7 Of course, any positive coefficient may be entered in the objective function and this is commendable, to scale the price level. As is, by the main theorem of linear programming, the factor input shadow prices fulfill $\mathbf{w} \cdot \mathbf{N}=\gamma$. Since $\gamma$ is of the order one but $\mathbf{N}$ of the order millions, $\mathbf{w}$ will be tiny. A handy objective function is $\mathbf{e} \cdot \mathbf{f} c$, where $\mathbf{e}$ is the unit vector with all components one. The dual constraints then show that $\mathbf{p} \cdot \mathbf{f}=\mathbf{e} \cdot \mathbf{f}$ and $\mathbf{w} \cdot \mathbf{N}=\mathbf{p} \cdot \gamma \mathbf{f}$. In other words, the product prices are normalized at unity and the factor input prices fulfill the potential national income identity. The proof is as follows. Multiplication of the dual constraint associated with variable $\mathbf{s}$, by $\mathbf{s}$, yields $\mathbf{p} \cdot\left(\mathbf{U}-\mathbf{V}^{\top}\right) \mathbf{s}+\mathbf{w} \cdot \mathbf{L s}=0$. Replace the two terms using the two respective constraints of program (14): $-\mathbf{p} \cdot \gamma \mathbf{f}+\mathbf{w} \cdot \mathbf{N}=0$, where (priced) inequalities are binding according to the phenomenon of complementary slackness. The product price normalization follows by the main theorem of linear programming or $\mathbf{w} \cdot \mathbf{N}=\mathbf{e} \cdot \mathbf{f} \gamma$.
}

\section{Relationship with the Solow residual}

This section is the centerpiece of the paper. The DebreuDiewert coefficient of resources and a generalized Solow residual are tied up into TFP growth. What is productivity?

An economy transforms physical resources into final consumption by means of production. The ratio of consumption to the resources is called the productivity of the economy. A multi-input and output measure is the Malmquist index proposed by Caves, Christensen, and Diewert (1982). Productivity may grow because the production possibility set increases or because resources are better utilized. Productivity growth equals the sum of technical change and efficiency change. Technical change is the shift of the production possibility frontier and efficiency change is the increase in the coefficient of resource utilization. The two add to productivity growth according to Nishimizu and Page (1982), Färe et al. (1994), and ten Raa and Mohnen (2002). I will now uncover the relationship at the level of generality of the Debreu model.

The point of departure is the Debreu-Diewert coefficient of resource utilization $\left(\rho^{*}\right)$, determined by program (7) or, using the Lemma, ${ }^{8}$

$\rho^{*}=\min \left\{r \mid \sum \mathbf{x}_{i}^{0}+\mathbf{y} \leqq r \mathbf{z}^{0}, \mathbf{y} \in Y\right\}$

Assuming free disposal, input may be added to $\mathbf{y} \in Y$ until the constraint is binding:

$\sum \mathbf{x}_{i}^{0}+\mathbf{y}=\rho^{*} \mathbf{z}^{0}$

This is the material balance.

Let $\mathbf{p}$ support the tight better set defined in (5), $\mathcal{B}^{*}$, in the sense introduced before (2). ${ }^{9}$ According to the phenomenon of complementary slackness, non-linear program (10) yields ${ }^{10}$

$\mathbf{p} \cdot \sum \mathbf{x}_{i}^{0}=\rho^{*} \mathbf{p} \cdot \mathbf{z}^{0}-\mathbf{p} \cdot \mathbf{y}$

This is the identity between national product and national income; it holds even when there is no free disposal and, therefore, the material balance, (11), is not fulfilled. The national product is on the left hand side and on the right hand side is factor income plus profit. (Remember, $\mathbf{y}$ is net input, hence $-\mathbf{y}$ is net output.) All this is at the optimum allocation $\left(\sum \mathbf{x}_{i}^{0}, \mathbf{y}, \rho^{*} \mathbf{z}^{0}\right)$ and supporting (or competitive) prices $\mathbf{p}$, not the actual allocation $\left(\sum \mathbf{x}_{i}^{0}, \mathbf{y}^{0}, \mathbf{z}^{0}\right)$ and prices.

The economy transforms resources $\mathbf{z}^{0}$ into consumption $\sum \mathbf{x}_{i}^{0}$. The ratio of the latter to the former constitutes the

\footnotetext{
${ }^{8}$ See the proof of the corollary.

${ }^{9}$ Footnote 4 shows that the supporting prices are not necessarily positive

10 The nonlinearity is due to production set $Y$.
} 
level of TFP. Since the objects are vectors, they must weighed by prices, for which $\mathbf{p}$ is employed. The level of TFP is thus $\mathbf{p} \cdot \sum \mathbf{x}_{i}^{0} / \mathbf{p} \cdot \mathbf{z}^{0}$. If there are constant returns to scale, profit is zero, and, by Eq. 12:

$\mathbf{p} \cdot \sum \mathbf{x}_{i}^{0} / \mathbf{p} \cdot \mathbf{z}^{0}=\rho^{*}$

This equation shows that the level of TFP is equal to the Debreu-Diewert coefficient of resource utilization.

Another interesting connection is the following. Let all variables vary with time and let $d$ denote a time derivative. Total factor productivity growth is the rate of growth of the level of TFP at fixed price weights: ${ }^{11}$

$T F P=\mathbf{p} \cdot d \sum \mathbf{x}_{i}^{0} / \mathbf{p} \cdot \sum \mathbf{x}_{i}^{0}-\mathbf{p} \cdot d \mathbf{z}^{0} / \mathbf{p} \cdot \mathbf{z}^{0}$

The following proposition shows that TFP is the sum of the Solow residual, generalized to Debreu's framework, and the rate of growth of the Debreu-Diewert coefficient. The generalized Solow residual is defined by

$S R=-\mathbf{p} \cdot d \mathbf{y} / \mathbf{p} \cdot \sum \mathbf{x}_{i}^{0}$

This expression features the change in optimal net output, $-\mathbf{y}$, and will be shown to be a generalized Solow residual indeed, evaluated at the frontier. The demonstration is in the next section, where the residual will be shown to measure the shift of the production possibility function.

Proposition Under constant returns to scale, $T F P=S R+d \rho * / \rho^{*}$.

Proof Under constant returns to scale Eq. 13 holds. Substitution in Eq. 14 yields $T F P=\left[\mathbf{p} \cdot d \sum \mathbf{x}_{i}^{0}-\rho^{*} \mathbf{p}\right.$. $\left.d \mathbf{z}^{0}\right] / \mathbf{p} \cdot \sum \mathbf{x}_{i}^{0}$. Substitution of the material balance, (11), and the product rule yield $T F P=\left[-\mathbf{p} \cdot d \mathbf{y}+\mathbf{p} \cdot \mathbf{z}^{0} d \rho^{*}\right] /$ $\mathbf{p} \cdot \sum \mathbf{x}_{i}^{0}$. Substitution of Eqs. 13 and 15 yields the posted formula. QED

The first TFP term, SR, reflects technical change. The second TFP term, $d \rho^{*} / \rho^{*}$, is the rate of growth of the Debreu-Diewert coefficient of resource utilization and, therefore, represents efficiency change.

\section{The Solow residual}

Solow (1957) divides commodities between a single output and factor inputs. Denoting the latter by a vector $\mathbf{l}$, the producible output is $F(\mathbf{l}, t)-s$, where $F(\cdot, t)$ is the production function at time $t$ (presumed quasi-concave) and $s$

\footnotetext{
11 Warning: I use TFP for TFP growth. No symbol is needed for the level of TFP.
}

is slack. ${ }^{12}$ A net input vector $\mathbf{y} \in Y$ has components $-F(\mathbf{l}, t)+s$ and $\mathbf{l}$, respectively. The production possibility set $Y$ is obtained by letting $\mathbf{I} \geqq \mathbf{0}$ and $s \geqq 0$. The vector of available resources, $\mathbf{z}^{0}$, has components 0 and $\mathbf{l}^{0}$, respectively. Let $\rho^{*}$ be the Debreu-Diewert coefficient of resource utilization and $\mathbf{y}$ be the optimal net input vector, which solve efficiency program (10), then $\mathbf{y}$ has components $-F(\mathbf{l}, t)$ and $\mathbf{l}=\rho^{*} \mathbf{l}^{0}$. The first or product component of the material balance, (11), reads

$$
\left(\sum \mathbf{x}_{i}^{0}\right)_{1}-F(\mathbf{l}, t)=\rho^{*} 0=0
$$

The other or factor components read

$0+\mathbf{l}=\rho^{*} \mathbf{l}^{0}$

An intuitive interpretation of the Debreu-Diewert coefficient of resource utilization, $\rho^{*}$, is in terms of actual output, $F\left(\mathbf{l}^{0}, t\right)-s^{0}$, where $F\left(\mathbf{l}^{0}, t\right)$ is potential output and $s^{0}$ is observed slack. Actual output could also be generated by optimal factor input I (with no slack). It follows that the actual/potential output ratio is $F(\mathbf{l}, t) / F\left(\mathbf{l}^{0}, t\right)$. By Eq. 17, this is $\rho^{*}$ if the production has constant returns to scale. The Debreu-Diewert coefficient is the ratio of actual to potential output.

As is well known, the solution $\mathbf{y}$ is supported by price vector $(1 \mathbf{w})=\left(1 \partial_{\mathbf{l}} F(\mathbf{l}, t)\right)$ where $\partial$ denotes partial derivatives (with respect to $\mathbf{I}$ in this case) or marginal productivities. ${ }^{13}$ I will now evaluate $S R=-\mathbf{p} \cdot d \mathbf{y} / \mathbf{p}$. $\sum \mathbf{x}_{i}^{0}$ of definition (15) for this special setting. The numerator reduces to

$-\mathbf{p} \cdot d \mathbf{y}=d F(\mathbf{l}, t)-\mathbf{w} d \mathbf{l}$

and the denominator is, in view of the first terms of (16) and (17),

$\mathbf{p} \cdot \sum \mathbf{x}_{i}^{0}=\left(\sum \mathbf{x}_{i}^{0}\right)_{1}+0=F(\mathbf{l}, t)$

using (16). Hence the quotient is

$$
\begin{aligned}
S R & =-\mathbf{p} \cdot d \mathbf{y} / \mathbf{p} \cdot \sum \mathbf{x}_{i}^{0} \\
& =d F(\mathbf{l}, t) / F(\mathbf{l}, t)-\sum_{k}\left[w_{k} l_{k} / F(\mathbf{l}, t)\right] d l_{k} / l_{k}
\end{aligned}
$$

The expression on the right hand side is, indeed, the residual between the output growth rate and the input growth rates, where value shares weight the latter. The shares add up if the production function has constant returns to scale, by Euler's theorem. The input prices are competitive marginal productivities, which are high in the sense that they leave no room for profit. The use of lower, observed prices, will bias upwardly expression (20).

\footnotetext{
${ }_{12}$ Slack scalar $s$ should not be confused with allocation vector $\mathbf{s}$ of Sect. 5.

${ }^{13}$ If $F(\cdot, t)$ is not differentiable, a subgradient will do.
} 
The main point of Solow (1957) was that the residual measures technical change, a result that is easily verified. By Eq. $18,-\mathbf{p} \cdot d \mathbf{y}=d F(\mathbf{l}, t)-\partial_{\mathbf{l}} F(\mathbf{l}, t) d \mathbf{l}$. But by total differentiation, $\quad d F(\mathbf{l}, t)=\partial_{\mathbf{l}} F(\mathbf{l}, t) d \mathbf{l}+\partial_{t} F(\mathbf{l}, t)$. Hence $S R=-\mathbf{p} \cdot d \mathbf{y} / \mathbf{p} \cdot \sum \mathbf{x}_{i}^{0}$ of definition (15) has a numerator $\partial_{t} F$ and we obtain, using Eq. 19,

$S R=-\mathbf{p} \cdot d \mathbf{y} / \mathbf{p} \cdot \sum \mathbf{x}_{i}^{0}=\partial_{t} F(\mathbf{l}, t) / F(\mathbf{l}, t)$

The Solow residual measures the relative shift of the production function indeed.

Residual expression (20) can be generalized to multiproducts. Then the output growth term is an output-value share weighted expression. Intermediate products can also be accommodated; this will be detailed in the next section. All are encompassed by definition (15): $S R=-\mathbf{p} \cdot d \mathbf{y} /$ $\mathbf{p} \cdot \sum \mathbf{x}_{i}^{0}$, where $-\mathbf{y}$ is resource minimizing net output and $\sum \mathbf{x}_{i}^{0}$ is observed total consumption.

For constant returns to scale, the minimization of resources subject to total consumption-see program (10)_amounts to a maximization of consumption subject to available resources_program (9). As was shown there, this merely involves a change of variable from $\mathbf{y}$ to $c \mathbf{y}$ and a replacement of $\sum \mathbf{x}_{i}^{0}$ by $c \sum \mathbf{x}_{i}^{0}$. The expansion factors $c$ in the numerator and in the denominator of the generalized Solow residual, $S R=-\mathbf{p} \cdot d \mathbf{y} / \mathbf{p} \cdot \sum \mathbf{x}_{i}^{0}$, cancel and its expression may therefore be reinterpreted in terms maximal consumption and sustaining optimal net output. The maximum consumption vector has the same proportions as the observed consumption vector. The prices in the generalized Solow residual are not affected at all, because of the constant returns to scale.

\section{Productivity and efficiency decompositions}

There are two further decompositions of TFP growth than in technical change and efficiency change. The first decomposition is in factor productivity growth rates; it sounds dull, but is not achieved in frontier analysis. The second decomposition is by input-output sector.

The decomposition by factor is standard neoclassical analysis, at least for the Solow residual. Assume constant returns to scale, then $\mathbf{p} \cdot \mathbf{y}=0$ and the generalized Solow residual becomes

$S R=-\mathbf{p} \cdot d \mathbf{y} / \mathbf{p} \cdot \sum \mathbf{x}_{i}^{0}=d \mathbf{p} \cdot \mathbf{y} / \mathbf{p} \cdot \sum \mathbf{x}_{i}^{0}$

Remember, $\mathbf{y}$ is the vector of net inputs. $\mathbf{p}$ is the vector of shadow prices or marginal productivities. Equation 22 imputes the technical change term of TFP to the various inputs. It is very general. It reduces to the more familiar Jorgenson and Griliches (1967) form in the Solow world with a macro-economic production function, introduced in the previous section. There $\mathbf{y}$ has components $-F(\mathbf{l}, t)$ and $\mathbf{l}$, and $\mathbf{p}=(1 \mathbf{w})=\left(1 \partial_{\mathbf{l}} F(\mathbf{l}, t)\right)$. Hence the numerator of the generalized Solow residual (22) reduces to $d \mathbf{p} \cdot \mathbf{y}=$ $d \mathbf{w} \cdot \mathbf{l}$, while the denominator is $F(\mathbf{l}, t)$ by Eq. 19 .

It follows that the Solow residual becomes

$S R=-\mathbf{p} \cdot d \mathbf{y} / \mathbf{p} \cdot \sum \mathbf{x}_{i}^{0}=\sum_{k}\left[w_{k} l_{k} / F(\mathbf{l}, t)\right] d w_{k} / w_{k}$

The expression on the right hand side is the growth rate of the factor productivity, with components weighted by their value shares. The input prices are competitive marginal productivities, which are high in the sense that they leave no room for profit. The use of lower, observed prices, will bias downward expression (23), unlike expression (20), which was biased upward in this case. The (primal) expression (20) and the (dual) expression (23) thus provide inconsistent estimates when no competitive prices are used.

The inclusion of efficiency change amounts to proportional increases of the factor productivity growth rates. By Debreu's equation (3), the minimally required physical resources, $\mathbf{z}$, are proportional to the utilizable physical resources, $\mathbf{z}^{0} .^{14}$ In the world of Solow this proportionality is between minimal factor inputs $\mathbf{l}$ and observed factor inputs $\mathbf{l}^{0}$, see Eq. 16 , or $\rho^{*}=l_{k} / l_{k}^{0}$, all $k$. Hence the efficiency change term of TFP reads, assuming constant returns to scale,

$$
\begin{aligned}
d \rho^{*} / \rho^{*} & =\sum_{k}\left[w_{k} l_{k} / F(\mathbf{l}, t)\right] d \rho^{*} / \rho^{*} \\
& =\sum_{k}\left[w_{k} l_{k} / F(\mathbf{l}, t)\right]\left(d l_{k} / l_{k}-d l_{k}^{0} / l_{k}^{0}\right)
\end{aligned}
$$

Substituting expressions (23) and (24) into the Proposition (Sect. 7), all TFP-growth is now decomposed in terms of factor contributions:

$T F P=\sum_{k}\left[w_{k} l_{k} / F(\mathbf{l}, t)\right]\left(d w_{k} / w_{k}+d l_{k} / l_{k}-d l_{k}^{0} / l_{k}^{0}\right)$

The leading term measures factor productivity growth and the remainder the factor utilization growth. For each factor, the value share of the factor weights the sum of the two growth measures.

The generalized Solow residual is decomposed by sector by adding the structure of Sect. 6. What follows is an activity variant of Hulten's (1978) analysis. In Sect. 6, the net input vector $\mathbf{y}$ sustaining maximal consumption has components $\left(\mathbf{U}-\mathbf{V}^{\top}\right) \mathbf{s}$ and $\mathbf{L s}$, where $\mathbf{U}$ is a table depicting the use of goods by sectors, $\mathbf{V}$ a table depicting the outputs of the sectors in terms of goods, $\mathbf{L}$ the factor input table, and allocation vector $\mathbf{s} \geqq 0$. Similarly, $\sum \mathbf{x}_{i}^{0}$ has components $\mathbf{f}$ and $\mathbf{0}$, where $\mathbf{f}$ is the vector of final goods consumption. The maximal consumption is $\gamma \mathbf{f}$, which we

\footnotetext{
${ }^{14}$ See the disclaimer in footnote 4 though.
} 
enter in the denominator, as discussed at the end of the last section. The respective prices are denoted $\mathbf{p}$ and $\mathbf{w}$, respectively; these are the shadow prices of program (9). The generalized Solow residual thus becomes

$$
S R=-\left\{\mathbf{p} \cdot d\left[\left(\mathbf{U}-\mathbf{V}^{\top}\right) \mathbf{s}\right]+\mathbf{w} \cdot d(\mathbf{L s})\right\} / \mathbf{p} \cdot(\gamma \mathbf{f})
$$

The shadow prices fulfill the dual constraint,

$\mathbf{p}^{\top}\left(\mathbf{U}-\mathbf{V}^{\top}\right)+\mathbf{w} \cdot \mathbf{L}-\boldsymbol{\sigma}^{\top}=\mathbf{0}$

where $\boldsymbol{\sigma}$ is the shadow price of $\mathbf{s} \geqq 0$. The product rule and substitution of Eq. 27 into expression (26) reduce the generalized Solow residual to

$S R=-\left[\mathbf{p}^{\top} d\left(\mathbf{U}-\mathbf{V}^{\top}\right) \cdot \mathbf{s}+\mathbf{w}^{\top} d \mathbf{L} \cdot \mathbf{s}+\boldsymbol{\sigma}^{\top} d \mathbf{s}\right] / \mathbf{p} \cdot(\gamma \mathbf{f})$

By the phenomenon of complementary slackness,

$\boldsymbol{\sigma} \cdot \mathbf{s}=0$

expression (28) becomes

$$
\begin{aligned}
S R= & \left(\mathbf{p}^{\top} d \mathbf{V}^{\top}-\mathbf{p}^{\top} d \mathbf{U}-\mathbf{w}^{\top} d \mathbf{L}+\operatorname{d} \boldsymbol{\sigma}\right)(\operatorname{diag} \mathbf{V} \mathbf{p})^{-1} \\
& \times(\operatorname{diag} \mathbf{V} \mathbf{p}) \mathbf{s} / \mathbf{p} \cdot(\gamma \mathbf{f})
\end{aligned}
$$

Here $\left(\mathbf{p}^{\top} d \mathbf{V}^{\top}-\mathbf{p}^{\top} d \mathbf{U}-\mathbf{w}^{\top} d \mathbf{L}+d \boldsymbol{\sigma}\right)(\operatorname{diag} \mathbf{V} \mathbf{p})^{-1}$ is the row vector of sectoral Solow residuals, while $(\operatorname{diag} \mathbf{V p}) \mathbf{s} / \mathbf{p} \cdot(\gamma \mathbf{f})$ is the vector of Domar weights, which add to the gross output/net output ratio of the economy, a number greater than one. ${ }^{15}$

To include sectoral efficiency changes, recall from Sect. 6 that the optimal (sustaining maximal consumption) and utilizable resources have only factor components, namely $\mathbf{L s}$ and $\mathbf{N}$, respectively. Application of the phenomenon of complementary slackness and the main theorem of linear programming to (9) yields $\mathbf{w}^{\top} \mathbf{L s}=\mathbf{w} \cdot \mathbf{N}=\gamma$. According to the Corollary, $\rho^{*}$ is the inverse of this expression. It follows that the efficiency term of TFP becomes, substituting in the denominator $\mathbf{w}^{\top} \mathbf{L s}=\mathbf{p}^{\top}\left(\mathbf{V}^{\top}-\mathbf{U}\right) \mathbf{s}=\mathbf{p} \cdot(\gamma \mathbf{f})$ by Eqs. 27, 29, and material balance (9), ${ }^{16}$

$$
\begin{aligned}
d \rho^{*} / \rho^{*} & =-d\left(\mathbf{w}^{\top} \mathbf{L s}\right) /\left(\mathbf{w}^{\top} \mathbf{L s}\right) \\
& =-\sum_{j}\left[d\left(\mathbf{w} \cdot \mathbf{l}_{. j} s_{j}\right) /\left(\mathbf{p} \cdot \mathbf{v}_{j} . s_{j}\right)\right] \cdot\left(\mathbf{p} \cdot \mathbf{v}_{j} \cdot s_{j}\right) / \mathbf{p} \cdot(\gamma \mathbf{f})
\end{aligned}
$$

where the summation is over sectors. The efficiency growth is a Domar weighted average of optimal factor input reduction growth rates.

\footnotetext{
15 This number is also called the Domar ratio. For any vector $\mathbf{x}, \operatorname{diag} \mathbf{x}$ denotes the diagonal matrix with $\mathbf{x}$ on the diagonal.

16 According to program (9), the material balance is an inequality. However, the premultiplication by the price vector eliminates the slack, by the phenomenon of complementary slackness. Alternatively, the material balance may be transformed to an equality in the same way that Eq. 11 was derived from program (10), assuming free disposal. Vectors $\mathbf{1}_{j}\left(\mathbf{v}_{j}\right)$ denotes the $j$-th column (row) of matrix $\mathbf{L}(\mathbf{V})$.
}

A further specification is that of input-output analysis, where $\mathbf{U}$ and $\mathbf{V}$ are square matrices, $\mathbf{V}^{\top} \mathbf{s}$ is denoted $\mathbf{q}$, the vector of (optimal) gross outputs, and $\mathbf{A}=\mathbf{U}\left(\mathbf{V}^{\top}\right)^{-1}$ and $\mathbf{F}=\mathbf{L}\left(\mathbf{V}^{\top}\right)^{-1}$ are the matrices of (intermediate and factor) input coefficients. Expression (26) for the generalized Solow residual becomes

$S R=-\{\mathbf{p} \cdot d[(\mathbf{A}-\mathbf{I}) \mathbf{q}]+\mathbf{w} \cdot d(\mathbf{F q})\} / \mathbf{p} \cdot(\gamma \mathbf{f})$

and price equation (27) reads

$\mathbf{p}^{\top}(\mathbf{A}-\mathbf{I})+\mathbf{w}^{\top} \mathbf{F}-\left(\mathbf{V}^{-1} \boldsymbol{\sigma}\right)^{\top}=\mathbf{0}$

Assume $\mathbf{s}>\mathbf{0} .{ }^{17}$ As shadow prices are nonnegative, Eq. 29 sets the last term of Eq. 33 zero:

$\mathbf{p}^{\top}(\mathbf{A}-\mathbf{I})+\mathbf{w}^{\top} \mathbf{F}=\mathbf{0}$

This permits the following rewrite of the generalized Solow residual, (32):

$S R=-\left(\mathbf{p}^{\top} d \mathbf{A}+\mathbf{w}^{\top} d \mathbf{F}\right)(\operatorname{diag} \mathbf{p})^{-1}(\operatorname{diag} \mathbf{p}) \mathbf{q} / \mathbf{p} \cdot(\gamma \mathbf{f})$

This is essentially formula (12) of Wolff (1994). ${ }^{18}$ The first half of this expression, $-\left(\mathbf{p}^{\top} d \mathbf{A}+\mathbf{w}^{\top} d \mathbf{F}\right)(\operatorname{diag} \mathbf{p})^{-1}$, is the row vector of sectoral Solow residuals and the remainder, $(\operatorname{diag} \mathbf{p}) \mathbf{q} / \mathbf{p} \cdot(\gamma \mathbf{f})$, is the vector of Domar weights, which add to the gross output/net output ratio of the economy, a number greater than one. ${ }^{19}$ Expression (35) details the right hand side of Eq. 21: The generalized Solow residual measures the shift of the production function by means of reductions in intermediate and factor input coefficients.

The inclusion of sectoral efficiency changes is analogous to Eq. 31, obtained by substitution of $\mathbf{L s}=\mathbf{F q}$, Eq. 34, and material balance $(\mathbf{I}-\mathbf{A}) \mathbf{q}=\gamma \mathbf{f}::^{20}$

$$
\begin{aligned}
d \rho^{*} / \rho^{*} & \left.=-d\left(\mathbf{w}^{\top} \mathbf{F} \mathbf{q}\right) /\left(\mathbf{w}^{\top} \mathbf{F q}\right)=-d\left[\mathbf{p}^{\top}(\mathbf{I}-\mathbf{A}) \mathbf{q}\right)\right] / \mathbf{p} \cdot(\gamma \mathbf{f}) \\
& =-\sum_{k}\left\{d\left[\left(p_{k}-\mathbf{p} \cdot \mathbf{a} \cdot k\right) q_{k}\right] / p_{k} q_{k}\right\} \cdot\left(p_{k} q_{k}\right) / \mathbf{p} \cdot(\gamma \mathbf{f})
\end{aligned}
$$

where the summation is over commodities. The efficiency growth is a Domar weighted average of optimal factor input or value-added reduction growth rates.

\footnotetext{
17 Well known sufficient conditions are $\mathbf{f}>\mathbf{0}$ and $\mathbf{A}$ has nonnegative Leontief inverse. For details see ten Raa (2005, Chapter 2).

${ }^{18}$ Wolff (1994) substitutes observed values for gross output $\mathbf{q}$ and final goods consumption $\gamma \mathbf{f}$, which are optimal. However, since gross output and final goods consumption are linked by the same Leontief inverse, $\mathbf{q}$ is obtained by inflating observed gross output by $\gamma$. As this factor cancels against the one in the denominator, the difference is immaterial.

19 The input-output disaggregation, (35), is slightly different than the activity analytic one, (30), as sectors are now defined in terms of products, but the totals are the same. This wedge disappears when secondary products are absent (in the sense that output table $\mathbf{V}$ is diagonal).

${ }^{20}$ See footnote 16.
} 


\section{Conclusion}

In this paper, I have interrelated the Debreu coefficient of resource utilization, the Solow residual, and TFP growth. Freed from individual data requirements, the Debreu coefficient's growth rate and the Solow residual sum to TFP growth. The procedure is equivalent to the imposition of Leontief preferences. The decomposition is the neoclassical counterpart to the decomposition of productivity growth into technical change and efficiency change made in frontier analysis and admits breakdowns by factor input as well as by sector.

Acknowledgments I gratefully acknowledge comments from Victoria Shestalova, Erwin Diewert, Rolf Färe, other participants of the Aggregation, Efficiency and Measurement Conference held on 23-25 May 2003 at the Department of Economics of University of California, Riverside, two anonymous referees and an anonymous associate editor.

Open Access This article is distributed under the terms of the Creative Commons Attribution Noncommercial License which permits any noncommercial use, distribution, and reproduction in any medium, provided the original author(s) and source are credited.

\section{References}

Caves DW, Christensen LR, Erwin Diewert W (1982) The economic theory of index numbers and the measurement of input, output, and productivity. Econometrica 50(6):1393-1414. doi: $10.2307 / 1913388$

Chakravarty SR (1992) Efficiency and concentration. J Prod Anal 3:249-255. doi:10.1007/BF00158355

Charnes A, Cooper WW, Rhodes E (1978) Measuring the efficiency of decision making units. Eur J Operat Res 2:419-444. doi: 10.1016/0377-2217(78)90138-8

Debreu G (1951) The coefficient of resource utilization. Econometrica 19(3):273-292. doi:10.2307/1906814

Diewert WE (1983) The measurement of waste within the production sector of an open economy. Scand J Econ 85(2):159-179. doi: $10.2307 / 3439476$

Diewert WE (2001) Which (old) ideas on productivity measurement are ready to use? In: Hulten CR, Dean ER, Harper MJ (eds) New developments in productivity analysis. University of Chicago Press, Chicago, pp 85-101

Färe R, Grosskopf S (1996) Productivity and intermediate products: a frontier approach. Econ Lett 50:65-70. doi:10.1016/0165-1765 (95)00729-6

Färe R, Shawna G, Norris M, Zhang Z (1994) Productivity growth, technical progress, and efficiency change in industrialized countries. Am Econ Rev 84(1):66-83

Färe R, Grosskopf S, Zelenyuk V (2002) Finding common ground: efficiency indices. UPEG working paper 0305. Presented at the North American productivity workshop at Union College, Schenectady, NY

Farrell MJ (1957) The measurement of productive efficiency. J Roy Stat Soc Ser A (Gen) 120(III):253-281. doi:10.2307/2343100

Førsund FR, Sarafoglou N (2002). On the origins of data envelopment analysis. J Prod Anal 17:23-40. doi:10.1023/A:1013519902012

Grifell-Tatjé E, Lovell CAK, Pastor JT (1998) A quasi-malmquist productivity index. J Prod Anal 10(1):7-20. doi:10.1023/A:1018 329930629

Hulten CR (1978) Growth accounting with intermediate inputs. Rev Econ Stud 45(3):511-518. doi:10.2307/2297252

Johansen L (1972) Production functions. North-Holland, Amsterdam Jorgenson DW, Griliches Z (1967) The explanation of productivity change. Rev Econ Stud 34(3):308-350. doi:10.2307/2296675

Kumar S, Robert Russell R (2002) Technological change, technological catch-up, and capital deepening: relative contributions to growth and convergence. Am Econ Rev 92(3):527-548. doi: $10.1257 / 00028280260136381$

Nishimizu M, Page JM Jr (1982) Total factor productivity growth, technological progress and technical efficiency change: dimensions of productivity change in Yugoslavia. Econ J 92:920-936. doi: $10.2307 / 2232675$

ten Raa T (2005) The economics of input-output analysis. Cambridge University Press, Cambridge

ten Raa T (2007) Don't aggregate efficiency but disaggregate inefficiency. In: Färe R, Grosskopf S, Primont D (eds) Aggregation, efficiency, and measurement. Springer, Heidelberg, pp 145-51

ten Raa T, Mohnen P (2002) Neoclassical growth accounting and frontier analysis: a synthesis. J Prod Anal 18:11-128

Solow RM (1957) Technical change and the aggregate production function. Rev Econ Stat 39(3):312-320. doi:10.2307/1926047

Wolff EN (1994). Productivity measurement within an input-output framework. Region Sci Urban Econ 24(1):75-92. doi:10.1016/ 0166-0462(94)90020-5

Zieschang KD (1984) An extended farrell technical efficiency measure. J Econ Theory 33(2):387-396. doi:10.1016/0022-0531 (84)90101-7 